\title{
ASLS - ein winkelstabiles Verriegelungsystem für Marknägel
}

\author{
Dankward Höntzsch
}

\section{Zusammenfassung}

Die Vorteile der winkelstabilen Verriegelung sind seit Langem bekannt, können bisher aber nur vereinzelt genutzt werden. Zum Beispiel sind die insertionsnahen Verriegelungsschrauben oder Klingen (DFN) bei vielen Nagelsystemen winkelstabil zu blockieren. Dies wird über die zentrale Verschlussschraube erreicht. So kann aber nur die insertionsnächste Schraube oder Klinge erreicht werden. Es gibt verschiedene Versuche, die Winkelstabilität durch Kunststoffinlays im Verriegelungsloch oder ein Gewinde passend zum Bolzengewinde im Verriegelungsloch $\mathrm{zu}$ erreichen. Allen bisherigen Systemen ist gemeinsam, dass neue und/oder Extramarknägel verwendet werden müssen. Bei winkelstabiler Verriegelung des Marknagels ist die relative Instabilität allein durch die Elastizität des Nagels und durch den Kontakt im Isthmus des Röhrenknochens bestimmt. Das „Wackeln“ der konventionellen Verriegelung ist dagegen unbestimmt und unkontrolliert. Wenn mehr Elastizität und Bewegung gewünscht wird, kann dies dann über einen dünneren Nagel erreicht werden, andererseits kann die Elastizität durch einen dickeren Nagel oder aufgebohrte Technik reduziert werden.

\section{ASLS - An Angle-Stable Locking System for Intramedullary Nails}

The advantages of angle-stable locking have been known for a long time but, as yet, could only be used in isolated cases. For example, the insertion-near locking screws or blades (DFN) can be blocked in an angle-stable manner in many nailing systems. This is achieved by a central locking screw. However, only the most insertion-near screws or blades can be reached in this way. There have been various attempts to achieve angle stability with plastic inserts in the locking hole or a thread in the locking hole adapted to the bolt's thread. A common feature of all previous systems is that new and/or additional intramedullary nails must be used. In angle-stability of the intramedullary nail the relative instability is determined solely by the elasticity of the nail and by the contact in the isthmus of the hollow bone. In contrast the free play of conventional locking is undetermined and uncontrolled. When more elasticity and movement are desired, this can be achieved by using a thinner nail, on the other hand, the elasticity can be reduced by using a thicker nail or a drilling out technique.

\section{Prinzip des ASLS-Systems}

Der Verriegelungsbolzen oder die Schraube (je nach Terminologie) trägt an der Spitze eine Kunststoffhülse aus resorbierbarem Kunststoff. Der Außendurchmesser dieser Kunststoffhülse entspricht im nicht expandierten Ruhezustand dem

OP-JOURNAL 2012; 28: 166-169

(C) Georg Thieme Verlag KG Stuttgart · New York DOI http://dx.doi.org/10.1055/s-0032-1315297
Innendurchmesser des Verriegelungslochs. Die Kunststoffhülse hat einen Kragen, welcher verhindert, dass die Kunststoffhülse durch das Verriegelungsloch im Nagel durchgedrückt wird (Abb. 1).

Mit dem 1. Bohrer werden mit der Stärke des Kerndurchmessers der Schraube gezielt (wie gewohnt) beide Kortikales durchbohrt. Die zugewandte Kortikalis wird auf den Innendurchmesser des Loches im Nagel $=$ Außendurchmesser der Hülse aufgebohrt.
Die Verriegelungsschraube mit der an der Spitze positionierten Kunststoffhülse wird durch das Loch der zugewandten Kortikalis soweit vorgeschoben, bis die Spitze der Schraube mit der Hülse im Verriegelungsloch des Nagels positioniert ist (Abb. 2).

Die Verriegelungsschraube hat im Bereich des Schaftes einen größeren Kerndurchmesser als an der Spitze. Dadurch wird bei weiterem Vorschrauben die Hülse zentripedal expandiert und gegen die Innenfläche des Loches des Marknagels gepresst. Es entsteht eine winkelstabile Blockade zwischen Marknagel und Verriegelungsschraube (Abb. 3).

Der Verriegelungsbolzen beim ASLS-System wird winkelstabil durch Spreizen eines Dübels, welcher genau ins Verriegelungsloch platziert wird.

Das erweiterte Loch in der zugewandten Kortikalis wird mit einem entsprechenden erweiterten Gewindeanteil der Verriegelungsschraube unter dem Schraubenkopf ausgefüllt (Abb. 3). Dieser Gewindesprung ist mit einer selbstschneidenden Nut versehen, sodass bei Kortikalisdicken, welche einen oder mehrere Gewindegänge benötigen, diese selbsttätig geschnitten werden.

Die Hülse ist aus resorbierbarem Polylactid. Es handelt sich um das bewährte Material, das für resorbierbare Fäden, Kordeln und Implantate verwendet wird. Untersuchungen haben gezeigt, dass die Winkelstabilität über mehrere Wochen oder gar Monate über $80 \%$ vom Ausgangswert aufrechterhalten wird. Die Hülse liegt im Nagel abgekapselt. Dieses resorbierbare Material wurde gewählt, um im Regelfall nicht entstehende, aber nicht ganz auszuschließende abgeriebene Partikel zu tolerieren. Dieses Polylactid hat auch die richtigen mechanischen Eigenschaften. Grundsätzlich und vom Prinzip könnte auch ein anderer biokompatibler geeigneter, Kunststoff verwendet werden. 


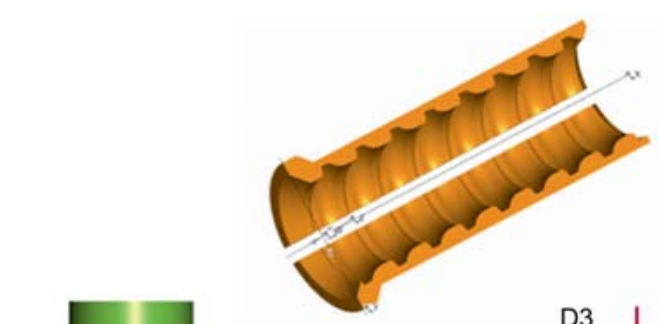

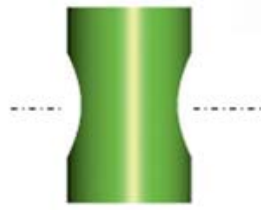
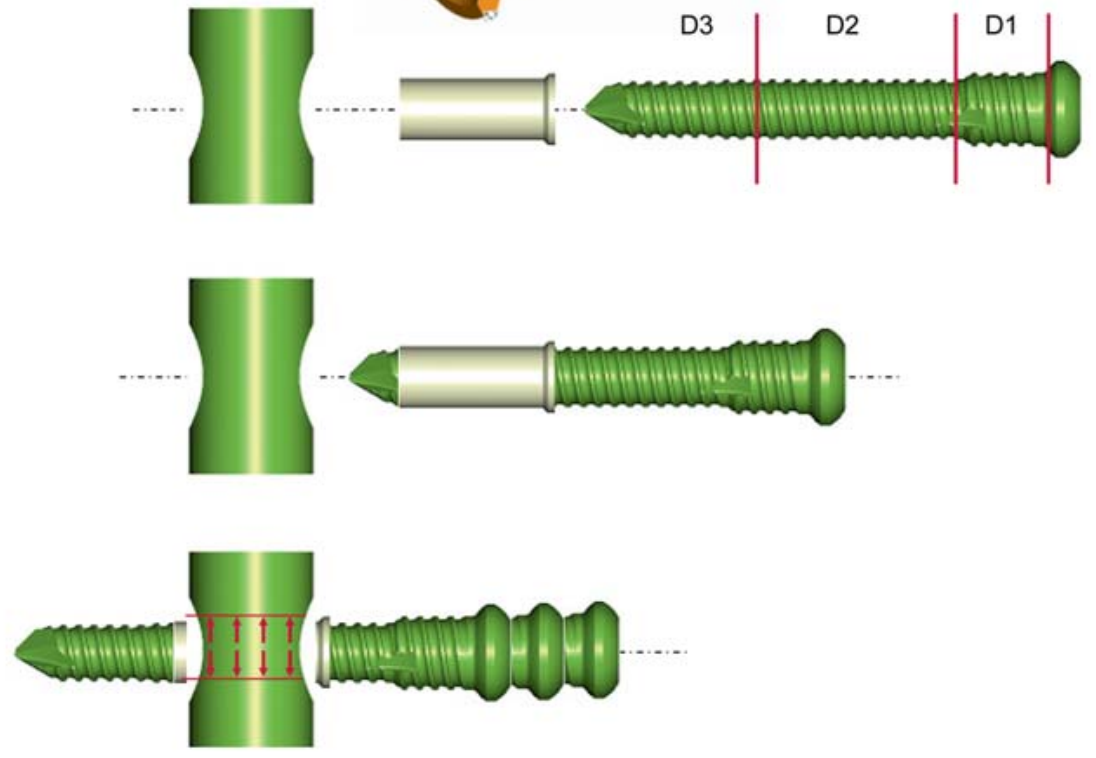

Abb. 1 Schematische Darstellung des ASLS-Systems (๔ Synthes GmbH).

Konventionelle Marknägel können verwendet werden. Während der Operation kann dann entschieden werden, ob konventionell oder winkelstabil verriegelt wird.

\section{Bisherige Erfahrungen}

Biomechanische Laborstudien haben gezeigt, dass die Stabilität auf axiale und Torsionsbelastung statisch und dynamisch deutlich höher ist als mit konven- tioneller Verriegelung. Versagenszustände treten später ein $[1,2,4]$.

Eine Studie mit Schafen als Versuchstieren hat gezeigt, dass durch die Marknagelung mit winkelstabiler Verriegelung (in dieser Studie Sondernägel mit Gewinde und Gewindeschrauben von Duda in Berlin) die Schafe früher frei bewegt und voll belastet haben. Die sekundäre Kallusbildung ist früher und homogener eingetreten [5].

Eine multizentrische Pilotstudie hat gezeigt, dass die Technik reproduzierbar und einfach ist. Komplikationen bei der Operationstechnik haben sich nicht ergeben [3].

Das ASLS-System ist in der Anwendung einfach. Nur 1 Schritt ist zusätzlich: das Überfräsen des zugewandten Loches. Die Lernkurve ist kurz.

In der Hand und Klinik des Autors bewährt sich die winkelstabile Verriegelung auch außerhalb dieser und einer derzeit laufenden prospektiv randomisierten Studie (Abb. 4). Signifikante Probleme in der Operationstechnik und mit der Technik des Verriegelungssystems zeigen sich in der Hand des Autors nicht. Andere Anwender berichten keine technischen Probleme. Wie groß die klinischen Vorteile endgültig sind, wird die weitere Anwendung in der Zukunft zeigen.

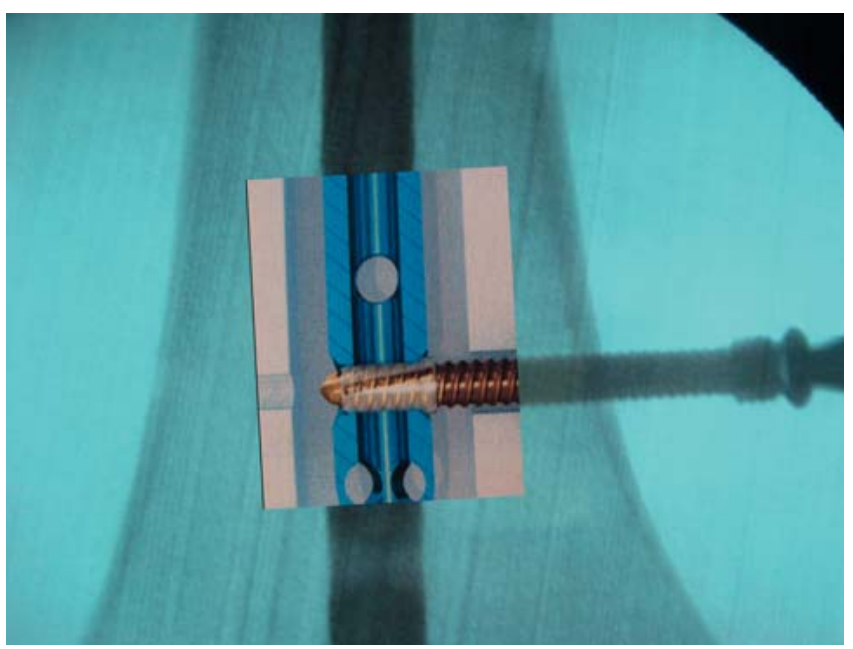

Abb. 2 Die Schraube wird mit dem aufgesetzten Schraubendreher durch das zugewandte Loch in der Knochenwand durchgeschoben, trifft das Loch des Verriegelungsmarknagels und wird dort so weit vorgeschoben, dass die Hülsenlippe auf dem Nagel aufsitzt. Diese Lippe verhindert, dass die Hülse zu weit durchgeschoben wird.

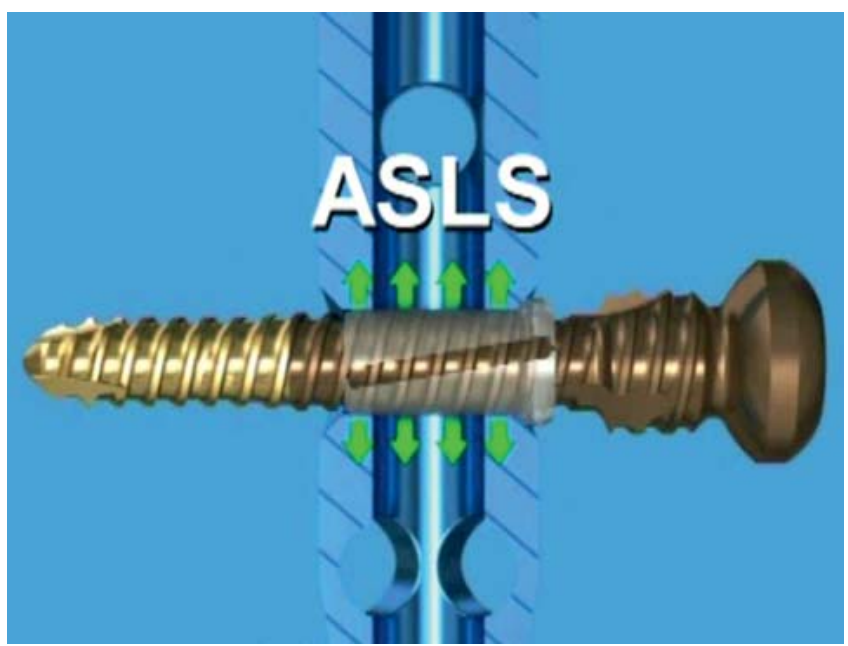

Abb. 3 Vordrehen der Schraube. Dadurch wird die Hülse expandiert. Man spürt dies an einem satten Drehmoment. Die Schraube durchquert das Loch in der Gegenkortikalis und taucht mit dem größeren Durchmesser unter dem Schraubenkopf in das Loch der zugewandten Kortikalis ein. In diesen Gewindesprung ist eine Schneidenut eingearbeitet, die, wenn notwendig, 1 bis 3 Gewindegänge in die zugewandten Kortikalis schneidet (@ Synthes $\mathrm{GmbH}$ ). 


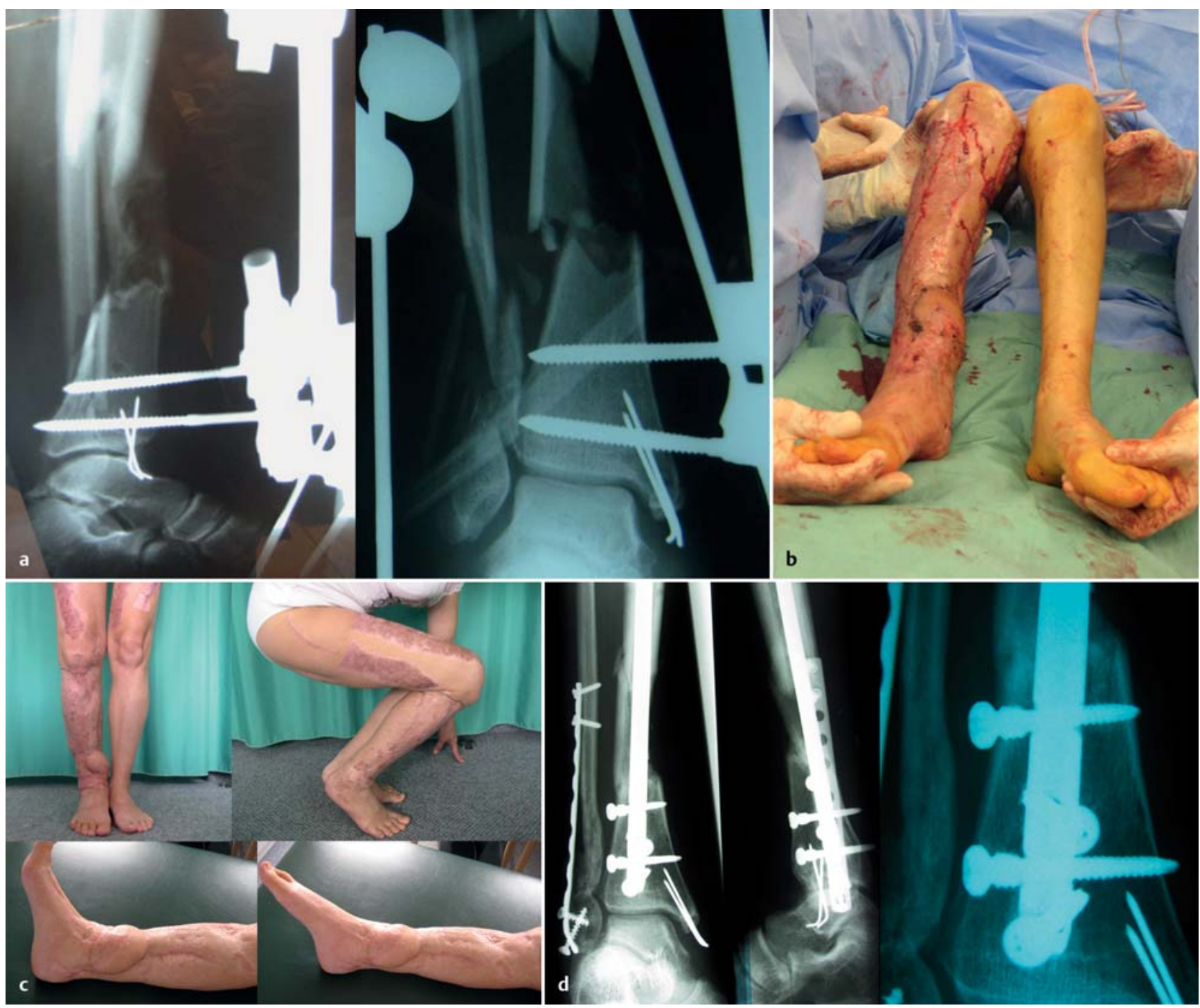

Abb. 4a bis d Ein Beispiel: a 54-jährige Frau mit III offener distaler Unterschenkeldefektfraktur, zunächst mit Fixateur externe und Weichteilsanierung versorgt; $\mathbf{b}$ früh sekundäre Marknagelung mit ETN und distal ASLS; c funktionelles Outcome und Belastungsmöglichkeit nach 6 Monaten gut; $\mathbf{d}$ bei knapper knöcherner Abstützung und medialem Defekt stützt der intramedulläre Marknagel mit winkelstabiler Verriegelung gut ab.

Die Indikation zur Marknagelung bzw. die Sicherheit bei Spezialindikationen konnte erweitert werden. Entsprechende Fälle sind dokumentiert und in Vorträgen gezeigt worden (Asal, Beil Schaser u.a.).

Die intraoperative Anwendung des ASLS-Systems ist einfach. Intra- und postoperative technische Probleme sind nicht beschrieben.

\section{Postoperative Behandlung}

Die postoperative Behandlung ist wie bei der Marknagelung mit konventioneller Verriegelung vorzunehmen. Dank der höheren Stabilität durch die winkelstabile Verriegelung kann früher und mit einer höheren Teilbelastung oder gar Vollbelastung begonnen werden.
Es erleichtert das Vorgehen „Belastung nach Vermögen“.

\section{Ergebnisse}

In einer prospektiven, multizentrischen Studie an 30 Patienten konnte gezeigt werden, dass die Operationstechnik einfach und reproduzierbar ist. Es gab kein technisches Versagen der Implantationstechnik [3].

Derzeit läuft eine multizentrische, prospektive, randomisierte Studie für distale Tibiafrakturen.

Um das Kriterium knöcherner Durchbau statistisch abzusichern, wäre eine sehr große Anzahl von Probanden notwendig.
Die statistisch geführte Fragestellung ist so gewählt, dass v.a. die Art der frühen Teil- und Vollbelastung und die Reaktion darauf überprüft werden. Hierzu führt der Unfallverletzte eine tägliche Belastung mit Waage und das Schreiben eines Tagebuchs durch. Engmaschig werden in allen ausgesuchten Zentren Belastungsanalysen mit identischen Systemen zur Ganganalyse und Belastung durchgeführt und dokumentiert (Zebris).

Die Rekrutierung der 140 Patienten ist abgeschlossen. Über die Ergebnisse kann noch nicht berichtet werden. Teilergebnisse sind aus studientechnischen Gründen noch nicht „geöffnet“ und dürfen auch nicht verwendet werden. Nur soweit zur Technik: Es ist zu keinem intraoperativen Versagen der ASLS-Technik 
gekommen. Rein technisch funktioniert das System gut und ohne Probleme.

Bisher sind keine kurz- oder langfristigen systembezogenen Komplikationen oder Nebenwirkungen eingetreten.

\section{Indikationen}

Nach heutigem Kenntnisstand ist die Indikation für das ASLS-System umso mehr gegeben, je relativer die Indikation für den Marknagel ist, obwohl er als zentraler Kraftträger wünschenswert ist.

- Je kleiner und instabiler das zu verriegelnde Fragment ist, desto eher ist die Indikation zur winkelstabilen Verriegelung des proximalen und/oder distalen Fragments angezeigt.

- Besonders profitieren erweiterte Marknagelindikationen.

- Indikation bei Femur, Tibia und Humersfrakturen.

- Indikation bei ante- und retrograden Nägeln.

- Gerade retrograd eingebrachte Nägel leiden häufig unter einem Wackeln des Nagels im Schaftbereich (Femur und Humerus). Das dort immer wieder beobachtete Wackeln des Nagels im Schaft kann nachhaltig abgestellt werden.

- Defektfrakturen

- Osteoporose
Die Indikation zur Marknagelung kann erweitert werden. Das ASLS-System macht die Verriegelung stabiler und belastbarer.

\section{Fazit}

Ein Vergleich mit der winkelstabil verriegelten Platte ist möglich. Die Platte wird als extramedullärer Fixateur interne beschrieben. Der winkelstabil verriegelte Marknagel (z.B. mit ASLS) kann analog als intramedullärer Fixateur interne bezeichnet werden.

Die Technik ist einfach und analog zur bekannten Arbeitsweise. Es ist nur ein einfacher Arbeitsschritt mehr notwendig, nämlich das größere Aufbohren der zugewandten Kortikalis. Die Technik und die Arbeitsschritte sind folglich kein Hindernis, die Technik anzuwenden. Die bisherigen Nagelsysteme können weiter verwendet werden. Hier ist keine Neuinvestition notwendig. Es kann intraoperativ entschieden werden, ob konventionell oder winkelstabil verriegelt werden soll. Die winkelstabile Verriegelung kann einseitig oder beidseitig, auch einseitig winkelstabil mit gegenseitiger dynamischer Verriegelung verwendet werden.

\section{Literatur}

${ }^{1}$ Gueorguiev B, Wähnert D, Albrecht D et al. Effect on dynamic mechanical stability and interfragmentary movement of angle-stable locking of intramedullary nails in unstable distal tibia fractures: a biomechanical study. J Trauma 2011; 70: 358-365

2 Gueorguiev B, Ockert B, Schwieger $K$ et al. Angular stability potentially permits fewer locking screws compared with conventional locking in intramedullary nailed distal tibia fractures: a biomechanical study. J Orthop Trauma 2011; 25: 340-346

${ }^{3}$ Höntzsch D, Blauth M, Attal R. [Angle-stable fixation of intramedullary nails using the Angular Stable Locking System ${ }^{\circledR}$ (ASLS)]. Oper Orthop Traumatol 2011; 23: 387-396

${ }^{4}$ Horn J, Linke B, Höntzsch D et al. Angle stable interlocking screws improve construct stability of intramedullary nailing of distal tibia fractures: a biomechanical study. Injury 2009; 40: 767-771

${ }^{5}$ Kaspar K, Schell H, Seebeck P. Angle stable locking reduces interfragmentary movements and promotes healing after unreamed nailing. Study of a displaced osteotomy model in sheep tibiae. J Bone Joint Surg [Am] 2005; 87: 2028-2037

Prof. Dr. med. Dankward Höntzsch Leitender Arzt

Abteilung für Medizin-Technische Entwicklungen BG-Unfallklinik Tübingen Schnarrenbergstraße 95 72076 Tübingen

hoentzsch@t-online.de 